\title{
Gravitational Collapse and Cosmic Censorship
}

\author{
Robert M. Wald \\ Enrico Fermi Institute and Department of Physics \\ University of Chicago \\ 5640 S. Ellis Avenue \\ Chicago, Illinois 60637-1433
}

February 5, 2008

\begin{abstract}
We review the status of the weak cosmic censorship conjecture, which asserts, in essence, that all singularities of gravitational collapse are hidden within black holes. Although little progress has been made toward a general proof (or disproof) of this conjecture, there has been some notable recent progress in the study of some examples and special cases related to the conjecture. These results support the view that naked singularities cannot arise generically.
\end{abstract}




\section{Introduction}

It has long been known that under a wide variety of circumstances, solutions to Einstein's equation with physically reasonable matter must develop singularities [1]. In particular, if a sufficiently large amount of mass is contained in a sufficiently small region, trapped surfaces must form [2] or future light cone reconvergence should occur [3], in which case gravitational collapse to a singularity must result. One of the key outstanding issues in classical general relativity is the determination of the nature of the singularities that result from gravitational collapse.

A key aspect of this issue is whether the singularities produced by gravitational collapse must always be hidden in a black hole- so that no "naked singularities", visible to a distant observer, can ever occur. The conjecture that, generically, the singularities of gravitational collapse are contained in black holes is known as the weak cosmic censorship conjecture [4]. (A much more precise statement of this conjecture will be given in Sec. 2 below.) A closely related conjecture, known as the strong cosmic censorship conjecture [5], asserts that, generically, timelike singularities never occur, so that even an observer who falls into a black hole will never "see" the singularity. This paper will focus exclusively on the weak cosmic censorship conjecture.

In the past seven years, there have been two articles in the New York Times indicating the demise of weak cosmic censorship [6]. The main theme of this paper is that such reports of the death of cosmic censorship are greatly exaggerated: Although very little progress has been made toward a general proof of the weak cosmic censorship conjecture, it remains in good health, and, indeed, is probably healthier today than at any time in the past.

\section{Formulation of the weak cosmic censorship conjecture}

The issue at the heart of the weak cosmic censorship conjecture can be expressed in graphic terms by posing the following question: Could a mad scientist - with arbitrarily large but finite resources - destroy the universe? We know that, in principle, such a mad scientist could produce a spacetime singularity by gathering together a sufficiently large amount of mass into a sufficiently small region. The essential content of the weak cosmic censorship 
conjecture is the assertion that, if he were to do so, neither the singularity he would produce nor any of its effects could ever propagate in such a way as to reach a distant observer. Of course, even if weak cosmic censorship fails, the universe might still be protected from destruction by mad scientists, since even if naked singularities were produced, they might always be of a benign character, with well defined rules - presumably arising from quantum gravity - governing dynamical evolution in their presence. However, if weak cosmic censorship fails, then in a literal sense, we would come face-to-face with the laws of quantum gravity whenever gravitational collapse to a naked singularity occurs in distant regions of our universe.

To formulate the statement of the weak cosmic censorship conjecture more precisely, we first need to make precise the notion of the "finiteness" of the resources available to our mad scientist. This notion is well modeled by restricting consideration to spacetimes containing an asymptotically flat initial data surface, i.e., a hypersurface $\Sigma$ on which the induced spatial metric, $h_{a b}$ becomes Euclidean at asymptotically large distances from some compact ("central") region, and the extrinsic curvature, $K_{a b}$, of $\Sigma$ goes to zero at a suitable rate at infinity. If matter fields are present, additional asymptotic fall-off conditions for the matter fields also would be required. (The precise asymptotic conditions on this initial data that would be most suitable to impose probably would best be left open until further progress is made in investigations of cosmic censorship, and will not be considered here.) In particular, it should be noted that the restriction to asymptotically flat initial data ensures that our mad scientist initially has only a finite total amount of energy at his disposal, but it does not place any direct restrictions on the initial conditions he might set up in the "central region" of the spacetime.

The basic idea of the weak cosmic censorship conjecture is that, starting from these initial conditions, any sufficiently distant observer will neither encounter any singularities nor any effects arising from-i.e., propagating out of - singularities. To make this idea more precise, we need a suitable notion of a spacetime being asymptotically flat "at large distances and at late times". Such a notion is provided by the requirement that the spacetime be asymptotically flat at future null infinity. The standard definition of asymptotic flatness at future null infinity requires that one be able to conformally embed the spacetime in a suitable way into a spacetime with a boundary, $\mathcal{I}^{+}$, which, roughly speaking, provides endpoints for the null geodesics which propagate to asymptotically large distances. The precise details of this definition are 
not crucial to our discussion and can be found in standard references (see, e.g., [四], [7]). The precise smoothness requirements most suitable to impose at $\mathcal{I}^{+}$undoubtedly will depend on the precise choice of asymptotic conditions on the initial data (see above), and will not be considered here.

For a spacetime, $\left(M, g_{a b}\right)$, which is asymptotically flat at future null infinity, the black hole region, $\mathcal{B}$, of the spacetime is defined by

$$
\mathcal{B}=M-I^{-}\left(\mathcal{I}^{+}\right)
$$

where the chronological past, $I^{-}$, is taken in the ("unphysical") conformally completed spacetime. The event horizon, $\mathcal{H}$, of the black hole is defined to be the boundary of $\mathcal{B}$ in $M$

$$
\mathcal{H}=\partial \mathcal{B}
$$

We are now in a position to give a relatively precise formulation of weak cosmic censorship:

Weak cosmic censorship conjecture: Let $\Sigma$ be a 3-manifold which, topologically, is the connected sum of $\mathcal{R}^{3}$ and a compact manifold. Let $\left(h_{a b}, K_{a b}, \psi\right)$ be nonsingular, asymptotically flat initial data on $\Sigma$ for a solution to Einstein's equation with suitable matter (where $\psi$ denotes the appropriate initial data for the matter). Then, generically, the maximal Cauchy evolution of this data is a spacetime, $\left(M, g_{a b}\right)$ which is asymptotically flat at future null infinity, with complete $\mathcal{I}^{+}$.

In this formulation of the conjecture, the asymptotic flatness of $\left(M, g_{a b}\right)$ (with complete $\mathcal{I}^{+}$) ensures that sufficiently distant observers can live out their lives in their entirety, free from the effects of any catastrophic events occurring in the central region of the spacetime. Furthermore, the fact that these observers lie in the domain of dependence of $\Sigma$ implies that they also are free from any non-deterministic effects that might occur if singularities are produced. If singularities are produced, they cannot be seen from $\mathcal{I}^{+}$.

The above conjecture remains somewhat imprecise on account of the two words written in italics. In order for the matter to be "suitable", it clearly is necessary that the coupled Einstein-matter field equations have a well posed initial value formulation. It undoubtedly also should be required that the matter stress-energy tensor satisfy suitable energy conditions, such as the dominant energy condition. In addition, it would seem reasonable to require that the matter be such that, in any fixed, globally hyperbolic, background 
spacetime (such as Minkowski spacetime), one always obtains globally nonsingular solutions of the matter field equations starting from regular initial data; otherwise, any "naked singularities" produced in the dynamical evolution of the Einstein-matter equations may have nothing to do with gravitational collapse. Note that this latter condition would rule out fluids as "suitable matter" (in particular, on account of "shell crossing" singularities and shocks), although fluid examples remain quite valuable as simple matter models for testing behavior related to cosmic censorship (see, e.g., [8]). It is not clear whether any further restrictions should be imposed-or, indeed, if some of the above restrictions should be weakened somewhat. In any case, the "suitable" matter fields certainly should include the Maxwell field and the Klein-Gordon scalar field.

The "generic" condition was inserted in the above conjecture because it would not be fatal to the physical content of the conjecture if examples exist where dynamical evolution produces naked singularities, provided that the initial data required for these examples is so special that it would be physically impossible to achieve. A way to express the idea that no generic violations of weak cosmic censorship occur would be to require that all initial data giving rise to violations of the behavior specified in the conjecture is confined to a "set of measure zero" and/or a "set whose closure has empty interior". Unfortunately, it is far from clear precisely what measure or topology should be imposed on the space of initial data. Undoubtedly, it will be necesary to develop a much deeper insight into the dynamics implied by Einstein's equation before a natural choice of measure or topology will emerge, and I feel that the precise definition of "generic" would best be left open until that point.

Does the weak cosmic censorship conjecture hold? To answer this question, we would need to know a great deal about the global properties of solutions to Einstein's equation. Global existence of solutions with nearly flat initial data has been proven [9]. Thus, weak cosmic censorship holds for nearly flat data - where the nonlinear effects of general relativity are too weak to produce any singularities at all. However, mathematical techniques have not progressed to a stage where a direct attempt at a general proof of the weak cosmic censorship conjecture would be feasible. Thus, the evidence both for and against the validity of weak cosmic censorship has been largely of an "anecdotal" or "circumstantial" nature, or has been confined to some very restricted cases (such as spherical symmetry). In the remaining sections 
of this paper, I shall briefly summarize much of this evidence.

\section{Some evidence in favor of cosmic censor- ship}

In this section, I shall describe some analyses in support of cosmic censorship. Most of the basic ideas described here are at least 20 years old, but some notable recent progress has occurred.

\subsection{Stability of black holes}

If weak cosmic censorship fails, then gravitational collapse can (generically) result in a naked singularity rather than a black hole. If so, then it is quite possible that the formation of a black hole would be a non-generic outcome of collapse. In that case, one might expect to see evidence of this in linear perturbation theory off of a background spacetime containing a black hole. Specifically, one might expect an initial, smooth perturbation to grow without bound on the black hole horizon, signaling the conversion of the black hole into a naked singularity. (In linear perturbation theory, a blow-up of the perturbation on the horizon could occur only at asymptotically late times, but it could occur at a finite time in the nonlinear theory.) Thus, the study of linear perturbations off of a black hole background provides an excellent testing ground for weak cosmic censorship. A demonstration of the linear instability of black holes would effectively disprove weak cosmic censorship, whereas a demonstration of their linear stability would provide some notable evidence in support of it.

The first analysis of the linear stability of the Schwarzschild black hole was given in 1970 by C.V. Vishveshwara [10], who established its stability to axial (i.e., "odd parity") perturbations. Shortly thereafter, a convincing demonstration of the stability of the Schwarzschild black hole - together with detailed information about the decay properties of the perturbations - was given by Price [11]. More recently, a completely rigorous proof of the boundedness of perturbations at asymptotically late times has been given 12.

The analysis of the stability of a Kerr black hole is much less tractable than the Schwarzschild case. Nevertheless, Whiting [13] succeeded in proving that no unstable modes exist. Thus, it appears that weak cosmic censorship 
has passed the crucial test of stability of black holes to general, linear perturbations.

Another test of stability can be performed for extremal charged Kerr black holes, i.e., charged Kerr black holes whose mass, $M$, angular momentum, $J$, and charge, $Q$, satisfy

$$
M^{2}=Q^{2}+(J / M)^{2}
$$

No stationary black hole solutions exist when the right side of Eq. (3) exceeds the left side. Thus, if one can get an extremal black hole to "swallow" an object whose charge and/or angular momentum is sufficiently large compared with its mass, there would be no black hole final state available for the system to settle down to. Presumably, a naked singularity then would result. However, an analysis of test particle motion in an extremal charged Kerr background indicates that it is not possible to get a black hole to swallow too much charge or angular momentum [14. Interestingly, a similar anaylsis of test particle motion for certain extremal "black hole" solutions of the Einstein-Maxwell equations with a positive cosmological constant-where the spacetime is asymptotically DeSitter rather than asymptotically flatindicates that "over-charging" can occur in this case [15]. However, these "black holes" are only rough analogs of black holes in asymptotically flat spacetimes; in particular, in these solutions there are naked singularities as seen from the DeSitter analog of $\mathcal{I}^{+}$. Even if these "black holes" could be "destroyed" by perturbations, this would not contradict the formulation of weak cosmic censorship given above for asymptotically flat spacetimesthough it would provide evidence against some formulations of strong cosmic censorship.

\subsection{Failed counterexamples}

A class of possible counterexamples to weak cosmic censorship involving collapsing shells of null dust was proposed by Penrose [16] and generalized by Gibbons 17] over 25 years ago. A similar class of possible counterexamples also can be given for time symmetric initial data. In the analysis of these classes of possible counterexamples, a key role is played by the following two results from the theory of black holes in general relativity (see, e.g., [1], [7]):

- If weak cosmic censorship holds, then every trapped surface must be entirely contained within a black hole. (Here, a trapped surface, $S$, is 
a compact, 2-dimensional surface having the property that the convergence of both the outgoing and ingoing null geodesics normal to $S$ is everywhere positive.)

- If weak cosmic censorship holds and if matter satisfies the null energy condition (i.e., if $T_{a b} k^{a} k^{b} \geq 0$ for all null $k^{a}$ ), then the area of the event horizon of a black hole cannot decrease with time.

Now consider a convex shell of null dust with flat interior, which collapses from infinity down to an infinite density singularity. (Note that such null dust presumably would not qualify as "suitable matter" in our formulation of the weak cosmic censorship conjecture, but if the inequality given below could be violated for null dust, it presumbly also could be violated for "suitable matter".) In this example, there are two free functions of two variables which may be specified arbitrarily, characterizing the initial "shape" and initial mass density of the shell. Except in the case of a spherical shell with constant mass density, the solution exterior to the shell is not known. Nevertheless, enough information about the solution can be deduced to make an interesting test of cosmic censorhip. Specifically, the Bondi mass, $M$, infinitesimally outside of the shell at past null infinity can be computed, and it seems reasonable to expect that there will exist exterior solutions with total ADM mass equal to $M$ (or, at least, arbitrarily close to $M$ ). Furthermore, by integrating the Raychaudhuri equation for outgoing null geodesic congruences across the delta-function mass distribution on the null shell one can determine the presence of trapped surfaces lying infinitesimally outside of the shell.

Suppose, now, that a trapped surface, $S$, lies infinitesimally outside of the shell, and let $A(S)$ denote its area. As mentioned above, if weak cosmic censorship holds, then $S$ must lie within a black hole. Let $A_{0}$ denote the area of the 2 -surface obtained by intersecting the event horizon, $\mathcal{H}$, of this black hole with the null shell. Then, assuming the validity of weak cosmic censorship, the following string of inequalities should hold:

$$
A(S) \leq A_{0} \leq 16 \pi M_{\mathrm{bh}}^{2} \leq 16 \pi M^{2}
$$

Here, the first inequality follows from the fact that $S$ lies within the black hole and the shell is infalling. The second inequality follows from the area nondecrease theorem (see above) together with the fact that the maximum possible 
area of a stationary black hole of mass $M_{\mathrm{bh}}$ is achieved by the Schwarschild value, $16 \pi M_{\mathrm{bh}}^{2}$. (Here it is assumed that the black hole settles down to a stationary final state.) The final inequality expresses the fact that the mass of the final black hole cannot exceed the total ADM mass of the spacetime.

Remarkably, the inequality $A(S) \leq 16 \pi M^{2}$ implied by Eq. (何) involves only quantities which can be computed without knowing the solution exterior to the shell, and thus can be readily checked. Failure of this inequality to hold in any example would be nearly fatal to cosmic censorship, as only a few small loopholes would remain - such as the possible "unsuitablity" of the null dust matter, the possibly "non-generic" nature of the example, and the (very remote) possiblity that the black hole does not become asymptotically stationary.

By making use of the fact that the interior of the shell is flat, the issue of whether the above inequality holds can be reduced to the issue of whether a certain "isoperimetric inequality" holds for (topological) spheres of non-negative mean curvature embedded in Euclidean 3-space. Until very recently, the inequality $A(S) \leq 16 \pi M^{2}$ had been proven only in some special cases. However, recent results of Trudinger 18 on strengthened isoperimetric inequalities has enabled a general proof to be given that $A(S) \leq 16 \pi M^{2}$ in all cases; see [19] for further discussion.

A similar argument - showing that an analog of (⿻1, must hold if weak cosmic censorship is valid - can be given for the case of time symmetric initial data (i.e., $K_{a b}=0$ ) on a spacelike hypersurface $\Sigma$. A minimal surface, $S$, on $\Sigma$ is a compact (without boundary) 2-surface on which $p \equiv h^{a b} p_{a b}=0$ everywhere, where $p_{a b}$ is the extrinsic curvature of $S$ in $\Sigma$. In the case where $K_{a b}=0$, both sets of null geodesics orthogonal to a minimal surface $S$ will have vanishing expansion, so $S$ will be marginally trapped. It then follows that $S$ must lie within a black hole. Now, let $S_{\text {out }}$ be the outermost minimal surface on $\Sigma$. Since the intersection of the black hole horizon, $\mathcal{H}$, with $\Sigma$ must lie outside of (or coincide with) $S_{\text {out }}$, and since $S_{\text {out }}$ is the outermost minimal surface, it follows that the area, $A_{0}$, of $\mathcal{H} \cap \Sigma$ cannot be smaller than $A\left(S_{\text {out }}\right)$. Hence, in analogy with Eq. (파), we obtain

$$
A\left(S_{\text {out }}\right) \leq A_{0} \leq 16 \pi M^{2}
$$

where $M$ denotes the ADM mass of the spacetime. Again, both $A\left(S_{\text {out }}\right)$ and $M$ can be calculated directly from the initial data given on $\Sigma$, without the 
necessity of evolving the data off of $\Sigma$. In essence, Eq. (5) shows that if cosmic censorship is valid, then a strengthened version of the positive mass theorem must hold for time symmetric initial data whenever minimal surfaces are present.

An argument for the validity of Eq. (5) was given in [20], but it relied on the assumed existence of particular foliation of $\Sigma$ by 2-surfaces, as first proposed by Geroch [21] in an argument for the positivity of total mass. However, very recently, a proof of existence of the required foliation has been given [22], thus establishing that Eq. (5) does, indeed, hold for general, time symmetric initial data.

Of course, the proof of the inequalities (4) and (5) for the very restricted classes of spacetimes to which they apply is a far cry from even the beginnings of a general proof of weak cosmic censorship. Nevertheless, it is very hard to understand why these highly nontrivial inequalities should hold unless weak cosmic censorship can be thought of as providing the underlying physical reason behind them. Thus, while it is not clear how much "ob-

jective evidence" in favor of cosmic censorship is provided by the failure of these counterexamples, their failure has given many researchers considerable confidence in the validity of weak cosmic censorship.

\section{The hoop conjecture}

Although the results of the previous section provide some suggestive evidence in favor of cosmic censorship, it is not surprising that not all researchers have been convinced by this evidence, and some serious doubts about the general validity of weak cosmic censorship have been expressed. Many of these doubts have centered upon an idea known as the hoop conjecture, which has been formulated as follows (see [23] or box 32.3 of [24]):

Hoop conjecture: Black holes with horizons form when and only when a mass $M$ gets compacted into a region whose circumference in EVERY direction is $\mathcal{C} \leq 4 \pi M$.

(Here, one envisions "passing a hoop" of circumference $4 \pi M$ around the matter in every direction to test this criterion.) The basic idea intended to be expressed by this conjecture is that gravitational collapse in all three spatial dimensions must occur in order for a black hole to form. If collapse occurs 
in fewer dimensions (i.e., to a 2-dimensional "pancake" configuration or a 1-dimensional "spindle" configuration), then the hoop conjecture is normally interpreted as asserting that a naked singularity should result.

Clearly, the above statement of the hoop conjecture in not intended to be mathematically precise, and there are some obvious mathematical difficulties with its formulation. Probably the most serious of these difficulties arises from the fact that the "only when" portion of the conjecture cannot be expected hold unless "gravitational energy" is included in the "mass $M$ ", since there should be no difficulty forming a black hole out of a sufficient concentration of gravitational radiation. However, there is no local notion of "gravitational energy" in general relativity. In addition, there is no obvious notion of the "circumference" a world tube in spacetime (such as the world tube containing the "mass $M$ " of the conjecture), since arbitrarily near (in spacetime) to any given 2-dimensional surface exterior to a world tube are 2-surfaces (approximated by suitably chosen broken null surfaces) with arbitraily small circumference in every direction. In other words, one can pass an arbitrarily small hoop around any concentration of mass by making segments of the hoop move in a suitable, relativistic manner as one moves the hoop around the mass. In order for the conjecture to have meaning, it is necessary to specify the choice of spacelike slicing on which the circumference is to be measured, but it is not obvious how this should be done.

I do not know how to overcome the difficulties in the formulation of the "only when" portion of the hoop conjecture. In the theorem of Schoen and Yau [2]-establishing the existence of trapped (or anti-trapped) surfaces in certain situations when a sufficiently large amount of matter is compacted into a sufficiently small region - the difficulties in defining the "circumference" are avoided by using the internal geometry on a spacelike slice to measure the "size" of the region. (This measure of the size also can be made arbitrarily small by appropriate choices of slicing, but the matter energymomentum density on the slice is then necessarily affected in a corresponding manner.) It should be noted that the theorem of Schoen and Yau does not actually establish a version of the "when" half of the hoop conjecture unless one assumes weak cosmic censorship, since the presence of a trapped surface necessarily implies the existence of a black hole only under that assumption (see the beginning of subsection 3.2).

The main motivation for the "when" half of the hoop conjecture appears to have arisen from the study of the collapse of matter with cylindrical sym- 
metry (i.e., rotational symmetry about an axis together with translational symmetry along that axis). It has long been known that cylindrically symmetric fluids can collapse to singularities, but no trapped surfaces ever form [23], 25], and the singularities are "visible" from infinity. These examples do not directly provide counterexamples to weak cosmic censorship because the cylindrically symmetric spacetimes are not asymptotically flat in the required sense: The matter distribution and curvature extend to infinity along the axis, and even in the directions perpendicular to the axis, the metric approaches flatness too slowly. However, these examples might seem to suggest that weak cosmic censorship could be violated in the collapse of a very long but finite "spindle" of matter.

However, although cylindrically symmetric fluids can collapse to a singularity, it does not appear that such singularities occur generically or that they occur at all with "suitable matter". Specifically, for a dust cylindrical shell, an arbitrarily small amount of rotation causes a "bounce" [26]. Furthermore, it has been shown that in the vacuum and Einstein-Maxwell cases, no singularities whatsoever occur in cylindrical collapse [27]. Thus, even if it turns out that the collapse of a finite "spindle" of fluid matter can produce naked singularities similar to those which occur in the exactly cylindrical case, it seems unlikely that spindle collapse will produce naked singularities that satisfy either the "suitable matter" or "generic" provisions of the weak cosmic censorship conjecture.

Nevertheless, Shapiro and Teukolsky [28] performed numerical calculations of the collapse of highly prolate gas spheroids and found behavior which they interpreted as both supporting the hoop conjecture and providing likely counterexamples to the weak cosmic censor conjecture. In their calculations, they evolved a number of spacetimes describing collapsing gas spheriods using maximal time slicing. They found that when the spheroid was highly prolate, a singularity formed just exterior to the ends of the spheroid - at which point, of course, they could no longer continue their numerical evolution. They then searched for trapped surfaces on the maximal hypersurfaces and found that none were present. They interpreted this as indicating that the singularity might be naked.

As already indicated above, even if the singularity they found were shown to be naked, it would be far from clear that the "suitable matter" and "generic" provisions of the weak cosmic censor conjecture could be satisfied. However, it also should be emphasized that the absence of a trapped 
surface lying on their maximal slices in the portion of the spacetime that they constructed does not provide much evidence that the singularity they found is naked. Indeed, even in Schwarzschild spacetime, it is possible to choose a (highly non-spherically-symmetric) time slice which comes arbitrarily close to the singularity inside the black hole, and yet has no trapped surfaces contained within its past [29]. A good test of whether something like this might be occurring in the Shapiro-Teukolsky examples is provided by the Penrose-Gibbons spacetimes described above in subsection 3.2. One can choose the shell of null dust to be highly prolate and one can arrange the collapse of the shell so that - in the flat hyperplane slicing of the flat interior of the shell (the analog of maximal slicing in the Shapiro-Teukolsky examples) - the singularity occurs at the ends first. One may then analyze whether trapped surfaces occur just outside the null shell and, if so, where they are located. Examples can be given where trapped surfaces exist, but no trapped surface is wholly contained to the past of the "last hyperplane" prior to encountering the singularity [30]. These examples suggest that if a significantly larger portion of the Shapiro-Teukolsky spacetimes were constructed (which could be done by using different choices of time slicing), then trapped surfaces enclosing the singularity might be found. Thus, at present, there do not appear to be strong reasons to believe that the singularities found by Shapiro and Teukolsky are actually naked.

In summary, if weak cosmic censorhip holds, then the Schoen and Yau theorem [2] can be viewed as giving a precise statement and proof of a version of the "when" portion of the hoop conjecture - although, as pointed out to me by N. O'Murchadha, the applicability of this theorem is extremely restrictive. However, formidable difficulties would have to be overcome even to give a precise formulation of the "only when" portion of the hoop conjecture, since one would need a notion of the gravitational contribution to the "mass $M$ ". Furthermore, even if some version of the "only when" portion of the conjecture turns out to be valid, there need not be any conflict with weak cosmic censorship, since it could well be the case that if suitable matter fails to be sufficiently compacted in all three spatial directions, then, generically, no singularity forms. In any case, I am not aware of any results related to the hoop conjecture which cast a serious doubt on the validity of weak cosmic censorship. 


\section{The spherically symmetric Einstein-Klein- Gordon System}

As previously emphasized, a general analysis of the validity of cosmic censorship would appear to require a much greater mastery of the global properties of solutions to Einstein's equation than is presently achievable. Therefore, it is natural to focus attention on more tractable special cases. The assumption of spherical symmetry greatly simplifies the analysis of gravitational collapse, and, largely for that reason, has been widely studied. It should be kept in mind that there is no guarantee that phenomena found in spherically symmetric gravitational collapse are representative of general phenomena. In particular, a phenomenon which is generic under the restriction to spherical symmetry need not be generic when that restriction is removed. Nevertheless, it is instructive to explore the phenomena that occur in spherical gravitational collapse, and to determine to what degree cosmic censorship holds in that case.

By Birkhoff's theorem the gravitational field itself has no dynamical degrees of freedom in the spherical case, so it is essential to have matter degrees of freedom present. Spherical collapse has been most widely studied with fluid matter, particularly "dust", i.e, a perfect fluid with $P=0$. Numerous examples have been found where naked singularities occur (see, e.g., [31], [32]). However, most of these examples appear to be non-generic in character, and all of them appear to rely on properties of fluid matter that also would allow one to produce singularities during evolution in flat spacetime. In particular, the "shell crossing" and "shell focusing" naked singularities found in the collapse of dust matter appear to depend crucially on the ability to "aim" the dust so as to produce infinite density before the self-gravitation of the dust becomes large. Thus, in order to obtain more insight into the validity of cosmic censorship in the spherically symmetric case, it would appear to be necessary to study examples with a more "suitable" form of matter.

A suitable form of matter which provides an excellent testing ground for cosmic censorship is provided by a massless Klein-Gordon scalar field, $\phi$. The complete system of equations for the Einstein-Klein-Gordon system are

$$
\begin{gathered}
\nabla^{a} \nabla_{a} \phi=0 \\
G_{a b}=8 \pi\left[\nabla_{a} \phi \nabla_{b} \phi-\frac{1}{2} g_{a b} \nabla_{c} \phi \nabla_{c} \phi\right]
\end{gathered}
$$


When restricted to spherical symmetry, Eqs.([6) and (7) simplify greatly from the general case, but, as will be indicated further below, they still provide a rich dynamics.

In a series of papers, Christodoulou [33-37] has given a remarkably complete analysis of the singularities that can arise in spherically symmetric solutions to the Einstein-Klein-Gordon equations. Christodoulou considered evolution from initial data posed on a future null cone, $C_{0}^{+}$, with vertex on the world line $\Gamma$ corresponding to the center of spherical symmetry, $r=0$. (Here $r$ denotes the usual Schwarzschild radial coordinate defined by $4 \pi r^{2}=A$, where $A$ denotes the area of the orbit the rotation group.) The initial data on $C_{0}^{+}$can be characterized by the function

$$
\alpha \equiv \frac{d}{d r}(r \phi)
$$

which may be freely specified on $C_{0}^{+}$subject to asymptotic conditions and boundary conditions at $r=0$. In 35] Christodoulou showed that unique solutions of bounded variation (defined precisely in [35]) exist provided that the initial data is such that the function $\alpha$ is of bounded variation on $C_{0}^{+}$.

In [36] Christodoulou investigated the global behavior spherically symmetric Einstein-Klein-Gordon solutions which possess the following additional conformal symmetry: There exists a one-parameter group of diffeomorphisms (parametrized by $\lambda$ ) under which, for some constant $k$,

$$
g_{a b} \rightarrow \lambda^{2} g_{a b}, \quad r \rightarrow \lambda r, \quad \phi \rightarrow \phi-k \ln \lambda
$$

Such solutions will not be asymptotically flat, but the initial data for these solutions can be suitably "truncated" so as to yield asymptotically flat data, and consequently they are relevant for testing cosmic censorship. By analyzing these solutions, Christodoulou proved [36] that there exist choices of asymptotically flat initial data which evolve to solutions with a naked singularity. In these solutions, a singularity first forms at the origin and then propagates out to infinity along a (singular) future null cone, reaching $\mathcal{I}^{+}$ at a finite retarded time. Thus, $\mathcal{I}^{+}$is incomplete in the maximally evolved spacetime. The null cone singularity is of a rather mild type, in that the curvature remains bounded as one approaches the cone away from the vertex, although derivatives of the curvature blow up on the cone. Christodoulou also proved that there exist choices of asymptotically flat initial data which 
evolve to what he referred to as "collapsed cone singularities". The solutions with collapsed cone singularities can be thought of as describing black holes of vanishing mass which possess a singular event horizon. In these solutions, $\mathcal{I}^{+}$is complete - so the conditions of our formulation of the weak cosmic censorship conjecture are satisfied - but the singularity is not really hidden in a black hole and from infinity one can "see" events which are arbitrarily close to the singularity.円

Thus, the analysis of [36] established for the first time that naked singularities can arise by the gravitational collapse of "suitable matter".2 A great deal of insight into the circumstances under which naked singularities are produced was provided by Choptuik's [39] numerical investigations of the behavior of Einstein-Klein-Gordon solutions which are "just on the verge" of collapsing to a black hole. Choptuik considered various one-parameter families of initial data with the property that for small values of the parameter, the incoming scalar waves are weak and disperse back to infinity, whereas for large values of the parameter, the incoming scalar waves are strong and collapse to a Schwarzschild black hole. Choptuik then tuned the parameter to the "borderline value" where collapse first occurs, and used mesh refinement techniques to study the properties of the "borderline solution" near $r=0$ in detail. Remarkably, he found that for all of the one-parameter families he considered, the borderline solution always asymptotically approached a particular "universal solution". Furthermore, this universal solution was found to possess a discrete self-similarity, i.e., it admits a diffeomorphism (as opposed to a one-parameter group of diffeomorphisms) satisfying Eq. (9) with $k=0$. Neither the universality of the borderline collapse behavior nor the discrete self-similarity of the universal solution had been anticipated prior to Choptuik's analysis. Most importantly for the issue of cosmic censorship, the numerical investigations by Choptuik (confirmed by others [40]) indicated

\footnotetext{
${ }^{1}$ It should be noted that all of Christodoulou's examples of naked singularities arise from initial data on $C_{0}^{+}$of a low differentiability class (but, of course, with $\alpha$ of bounded variation, so that the initial value formulation is well posed). His examples with collapsed cone singularities can have initial data of arbitrarily high (but finite) differentiability.

${ }^{2}$ The existence of a naked singularity had previously been claimed for a particular scale invariant solution of the Einstein-Klein-Gordon equations (i.e., a solution satisfying Eq. (9) with $k=0$ ), which has been studied by a number of authors [38] (see also 35]). However, the analysis given in [36] shows that this solution (or, more precisely, suitable asymptotically flat "truncations" of this solution) actually corresponds to a collapsed cone singularity rather than a naked singularity.
} 
that the borderline solutions possess naked singularities of a nature similar to Christodoulou's examples.

Similar discrete or continuous self-similarity has been found to occur in the borderline solutions for a number of other systems (see, in particular, [41]). Presumably, these borderline solutions also possess naked singularities (although I am not aware of demonstrations of this). However, in some systems where there exist unstable, stationary, nonsingular solutions - in particular, in the Einstein-Yang-Mills system - some borderline solutions approach one of these stationary, nonsingular solutions rather than a self-similar solution [42].

The fact that naked singularities in the spherically symmetric EinsteinKlein-Gordon system were encountered in Choptuik's numerical calculations only for the borderline solutions suggests that the occurrence of naked singularities is non-generic. Clearly, no definitive conclusions in this regard can be drawn from numerical studies. However, an analytic demonstration of the non-generic character of naked singularities has recently been given by Christodoulou [37]. In order to state this result, it is useful to classify solutions arising from the maximal evolution of asymptotically flat initial data on $C_{0}^{+}$as follows:

case (i): No singularities at all occur at any finite advanced time and $\mathcal{I}^{+}$is future complete. This case necessarily arises when the initial data is sufficiently "small" 35.

case (ii): A singularity forms at a finite advanced time, but it is entirely contained within a black hole, as in the "standard picture" of gravitational collapse. In particular, $\mathcal{I}^{+}$is complete, and in order to reach the singularity, an observer must pass through a (non-singular) event horizon.

case (iii): Neither case (i) nor case (ii) holds.

Note that under this classification, case (iii) includes all solutions with naked singularities and collapsed cone singularities, as well as any solutions with

\footnotetext{
${ }^{3}$ In this case, the spacetime also must be future timelike and null geodesically complete, with the possible exception of the "central geodesic" $\Gamma$ at $r=0$, whose completeness was not explored by Christodoulou.
} 
other, as yet undiscovered, pathologies that might be viewed as contrary to the spirit of the weak cosmic censorship conjecture.

Christodoulou proved the following [37]: Consider any initial datacharacterized by the function $\alpha_{0}$-which evolves to a spacetime in category (iii) above. Then there exists a continuous function $f$ such that for any real number $c \neq 0$, the initial data characterized by $\alpha=\alpha_{0}+c f$ evolves to a spacetime in category (ii). In other words, by an arbitrarily small perturbation of the initial data, a spacetime containing a naked singularity (or a collapsed cone singularity or other pathology) can be converted to a black hole. Thus, within the class of initial data of bounded variation, solutions with naked singularities are non-generic in the above, precise sense.

Although restricted to the case of the spherically symmetric EinsteinKlein-Gordon equations, the above result provides the first true cosmic censorship theorem for a nontrivial system.

\section{Conclusions}

Although the question of whether weak cosmic censorship holds remains very far from being settled, there appears to be growing evidence in support of its validity. This evidence consists primarily of the stability of black holes (see subsection 3.1), the proof of the failure of certain classes of counterexamples (see subsection 3.2), and the proof of a cosmic censorship theorem for the spherically symmetric Einstein-Klein-Gordon system (see Sec. 5).

\section{Acknowledgements}

I wish to thank Demetrios Christodoulou for reading the manuscript. This research was supported in part by NSF grant PHY 95-14726 to the University of Chicago.

\section{References}

\footnotetext{
${ }^{4}$ The borderline solutions studied by Choptuik presumably comprise a surface, $\mathcal{S}$, of co-dimension 1 in the space of initial data. In order to have the above property at $\alpha_{0} \in \mathcal{S}$, Christodoulou's one parameter family must be tangent to $\mathcal{S}$, and $\mathcal{S}$ must be convex (as viewed from the "black hole side") in the direction defined by this family.
} 
[1] S.W. Hawking and G.F.R. Ellis, The Large Scale Structure of SpaceTime, Cambridge University Press (Cambridge, 1973).

[2] R. Schoen and S.-T. Yau, Commun. Math. Phys. 90, 575 (1983).

[3] R. Penrose, in Black Holes and Relativistic Stars, ed. R.M. Wald, University of Chicago Press (in press).

[4] R. Penrose, Revistas del Nuovo Cimento 1, 252 (1969).

[5] R. Penrose, in General Relativity, an Einstein Centennary Survey, ed. S.W. Hawking and W. Israel, Cambridge University Press (Cambridge, 1979)

[6] "Computer defies Einstein's theory", New York Times, March 10, 1991, section 1, P.21; "A Bet on a Cosmic Scale, and a Concession, Sort of", New York Times, February 12, 1997, section 1, P.1.

[7] R.M. Wald, General Relativity, University of Chicago Press (Chicago, 1984).

[8] P.S. Joshi and I.H. Dwivedi, Commun. Math. Phys. 146, 333 (1992).

[9] D. Christodoulou and S. Klainerman, The Global Nonlinear Stability of Minkowski Space, Princeton University Press (Princeton, 1993).

[10] C.V. Vishveshwara, Phys. Rev. D1, 2870 (1970).

[11] R. Price, Phys. Rev. D5, 2419 and 2439 (1972).

[12] B.S. Kay and R.M. Wald, Class. Quant. Grav. 4, 893 (1987).

[13] B.F. Whiting, J. Math. Phys. 30, 1301 (1989).

[14] R.M. Wald, Ann. Phys. 82, 548 (1974); I. Semiz Class. Quant. Grav. 7, 353 (1990).

[15] D.R. Brill, G.T. Horowitz, D. Kastor, and J. Traschen, Phys. Rev. D49, 840 (1994).

[16] R. Penrose, Ann. N.Y. Acad. Sci. 224, 125 (1973). 
[17] G. Gibbons, Commun. Math. Phys. 27, 87 (1972).

[18] N.S. Trudinger, Ann. Inst. Henri Poincare 11, 411 (1994).

[19] G. Gibbons, "Collapsing shells and the isoperimetric inequality for black holes", hep-th/9701049.

[20] P.S. Jang and R.M. Wald, J. Math. Phys. 18, 41 (1977).

[21] R. Geroch, Ann. N.Y. Acad. Sci. 224, 108 (1973).

[22] G. Huisken and T. Ilmanen, "Proof of the Penrose Inequality", to appear.

[23] K.S. Thorne, in Magic Without Magic: John Archibald Wheeler, ed. J. Klauder, W.H. Freeman (San Francisco, 1972).

[24] C.W. Misner, K.S. Thorne, and J.A. Wheeler, Gravitation, W.H. Freeman (San Francisco, 1973).

[25] P.T. Chrusciel, Ann. Phys. 202, 100 (1990).

[26] T.A. Apostolatos and K.S. Thorne, Phys. Rev. D46, 2435 (1992).

[27] B. Berger, P.T. Chrusciel, and V. Moncrief, Ann. Phys. 237, 322 (1995).

[28] S. Shapiro and S.A. Teukolsky, Phys. Rev. Lett 66, 994 (1991).

[29] V. Iyer and R.M. Wald, Phys. Rev. D44, 3719 (1991).

[30] K.P. Tod, Class. Quant. Grav. 9, 1581 (1992); K.P. Tod, unpublished; M. Pelath, unpublished.

[31] P. Yodzis, H.-J. Seifert, and H. Muller zum Hagen, Commun. Math. Phys. 34, 135 (1973).

[32] D.M. Eardley and L. Smarr, Phys. Rev. D19, 2239 (1979); D. Christodoulou, Commun. Math. Phys. 93, 171 (1984); P.S. Joshi and I.H. Dwivedi, Phys. Rev. D47, 5357 (1993).

[33] D. Christodoulou, Commun. Math. Phys. 109, 613 (1987). 
[34] D. Christodoulou, Commun. Pure \& Applied Math. XLIV, 339 (1991).

[35] D. Christodoulou, Commun. Pure \& Applied Math. XLVI, 1131 (1993).

[36] D. Christodoulou, Ann. Math. 140, 607 (1994).

[37] D. Christodoulou, Ann. Math. (in press).

[38] T. Maithreyan, Ph.D. thesis, Boston University, 1984 (unpublished); M.D. Roberts, Gen. Rel. and Grav. 21, 907 (1989); P.R. Brady, Class. Quant. Grav. 11, 1255 (1994); Y. Oshiro, K. Nakamura, and A. Tomimatsu, Prog. Theor. Phys. 91, 1265 (1994).

[39] M.W. Choptuik, Phys. Rev. Lett 70, 9 (1993).

[40] R.S. Hamade and J.M. Stewart, Class. Quant. Grav. 13, 497 (1996).

[41] A.M. Abrahams and C.R. Evans, Phys. Rev. Lett 70, 2980 (1993); C.R. Evans and J.S. Coleman, Phys. Rev. Lett 72, 1782 (1994); E.W. Hirschmann and D.M. Eardley, Phys. Rev. D51, 4198 (1995).

[42] M.W. Choptuik, T. Chmaj, and P. Bizon, Phys. Rev. Lett 77, 424 (1996). 